\title{
Minimal field requirement in precessional magnetization switching
}

\author{
Di Xiao* M. Tsoi, and Qian Niu \\ Department of Physics, The University of Texas at Austin, Austin, TX 78712
}

\begin{abstract}
We investigate the minimal field strength in precessional magnetization switching using the Landau-Lifshitz-Gilbert equation in under-critically damped systems. It is shown that precessional switching occurs when localized trajectories in phase space become unlocalized upon application of field pulses. By studying the evolution of the phase space, we obtain the analytical expression of the critical switching field in the limit of small damping for a magnetic object with biaxial anisotropy. We also calculate the switching times for the zero damping situation. We show that applying field along the medium axis is good for both small field and fast switching times.
\end{abstract}

PACS numbers: 75.60.Jk,75.75.+a

\section{INTRODUCTION}

Magnetization reversal in magnetic particles and thin films has been a continuous and growing topic in the past several decades, motivated by its great application potential in magnetic data storage and random access memories (RAM). Recent development of new fabrication techniques has made it possible to produce nanometersized magnetic objects with well-controlled shape, structure, and chemical composition. Magnetic anisotropy of these objects, including shape anisotropy and magnetocrystalline anisotropy, makes the dynamic magnetization processes highly nonlinear. A thorough understanding of micromagnetic dynamics is thus desirable as many efforts have already been made in this field ${ }^{1.2}$

In the past, magnetization reversal is realized by applying a magnetic field pulse mainly antiparallel to the initial magnetization. The magnetization will then undergo multiple rotations around the local effective field to reach the final equilibrium direction. This process is best viewed by drawing the energy landscape of the system. Assume magnetization in the $\hat{x}$ and $-\hat{x}$ directions gives the local energy minima. As shown in Fig. 1] upon application of the field pulse, the landscape is tilted, making the barrier disappear. The system will jump to the global minimum and the magnetization is therefore switched from one direction to another. It is clear that the applied field has to be strong enough to overcome the energy barrier. The critical switching field is first discussed in the uniaxial anisotropy case in the pioneering work by Stoner and Wahlfarth,,$\frac{3}{-}$ and recently extended to non-uniaxial and three dimensional cases, 4.5 Energy dissipation is necessary in this process for the system to move from one minimum to another. We call this type of reversal the Stoner-Wahlfarth (SW) type. Typical reversal time for such a process is of the order of nanoseconds.

Recently, a novel approach towards ultrafast magnetization switching by precessional motion has been proposed and observed experimentally ${ }^{6,7,8,9,10,11,12}$ Instead of applying a field pulse antiparallel to the initial magnetization, a perpendicular field pulse is applied and induces a large angle precession. If the pulse is termi- nated at $180^{\circ}$ angle of the precession, whose period is usually of the order of picoseconds, the magnetization is reversed. However, the effective reversal times could be several nanoseconds due to the decay time of residual magnetic precession ("ringing") if the magnetization does not end up in the final equilibrium state precisely. One can overcome this difficulty by fine tuning the pulse parameters so that the magnetization will move along the so-called ballistic trajectory, eliminating the ringing effect. 10 Thereby the fundamental ultrafast limit of field induced magnetization reversal is reached. In contrast to the SW type reversal, the precessional switching is so fast that dissipative effects can be neglected. In other words, the system energy is conserved and the problem can be formulated using the Hamiltonian equations.

Such a precessional switching opens a way to reduce not only the reversal time but also the field required for switching. As pointed out by early numer-

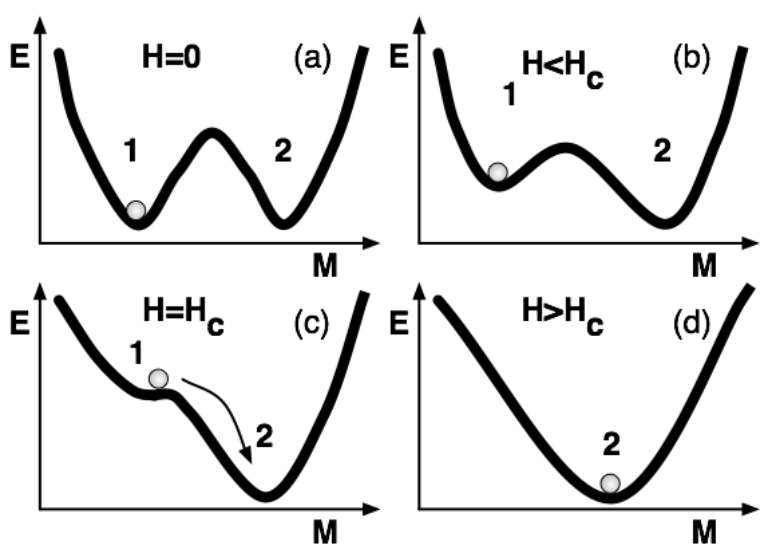

FIG. 1: Typical energy landscapes: energy $E$ vs magnetization $M$ at different value of applied field $H$. In (a) $H=0$, system sits on one of its local minimum 1 separated with 2 by a barrier. In (b) $H<H_{c}$, the critical field, position 1 is still a local minimum and magnetization reversal will not occur. In (c) $H=H_{c}$, the minimum 1 and the barrier coalesce, any fluctuation will cause the system jump to minimum 2. In (d) $H>H_{c}$, there is only one minimum, the magnetization is reversed. 


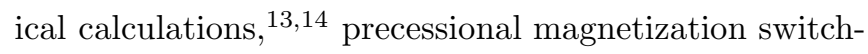
ing can be observed well below the Stoner-Wohlfart limit. This result is recently recovered by considering the precessional switching as a result of bifurcation, the longterm behavior of the dissipative system ${ }^{\underline{9}}$ However, to our knowledge, no systematic study of the minimal field strength exists in the literature and most of the work was done by numerical means.

In this paper we investigate the minimal field strength required for precessional switching of a magnetic object with biaxial anisotropy. We use phase space analysis as our main tool to tackle this problem. It turns out that the mechanism of the precessional switching is directly related to the evolution of the localized and unlocalized trajectories in the phase space. Unlike the SW type switching, where the fixed points and their movement are the central objects to study, in the precessional switching the states move along equienergy curves and we need follow their motion globally. The minimal (critical) field is obtained when the localized trajectories become unlocalized. By studying the phase space evolution we are able to obtain the analytical expression for the critical field. This result provides useful information on the design of field pulses for precessional switching based devices.

The paper is organized as follows. In Sec. II we present a simple model describing magnetization dynamics and define the central problem. We then in Sec. III consider the zero damping situation in which the switching field is applied perpendicular to the easy axis. We also calculate the switching times for this configuration. In Sec. IV with the assumption of very small damping we study the applied field perpendicular to the hard axis configuration and compare our result with the standard SW model. Finally the paper is summarized in Sec. $\mathrm{D}$

\section{MODEL}

The simplest micromagnetic model is the so-called macro-spin model, i.e., the magnetization is uniform and displays collective dynamics. This model is valid for small object size (reaching the single domain limit) and low magnetic coercivity. The motion of magnetization $\boldsymbol{M}$ with a phenomenological damping is governed by the Landau-Lifshitz-Gilbert (LLG) equation. This equation will be used in the following dimensionless form:

$$
\frac{\mathrm{d} \boldsymbol{m}}{\mathrm{d} t}=-\boldsymbol{m} \times \boldsymbol{h}_{\mathrm{eff}}+\alpha \boldsymbol{m} \times \frac{\mathrm{d} \boldsymbol{m}}{\mathrm{d} t} .
$$

Here $\boldsymbol{m}=\boldsymbol{M} / M_{s}$ is the magnetization unit vector, $\boldsymbol{h}_{\text {eff }}=\boldsymbol{H}_{\text {eff }} / M_{s}$ is the scaled effective field, time is measured in units of $\left(\gamma M_{s}\right)^{-1}, M_{s}$ is the saturation magnetization, $\gamma$ is the absolute value of the gyromagnetic ratio, and $\alpha$ is the dimensionless damping parameter.

We consider a very thin film in the $x-y$ plane with an in-plane uniaxial anisotropy, taking $x$ the easy axis of magnetization. The demagnetizing field factors are practically equal to zero in the film plane and one perpendicular to the film plane, respectively. The magnetic energy density is written

$$
w(\boldsymbol{m}, \boldsymbol{h})=\frac{1}{2} m_{z}^{2}-\frac{1}{2} K m_{x}^{2}-\boldsymbol{h} \cdot \boldsymbol{m},
$$

where $K>0$ accounts for the scaled in-plane $x$-axis anisotropy )and $\boldsymbol{h}$ denotes applied field. Usually the value of $K$ is about 0.01 for thin films because of the large demagnetizing field in the $\hat{z}$ direction. However, after proper scaling the above expression can be also used to describe the energy density of small magnetic particles with biaxial anisotropy as well. In following discussions we assume $K>0$.

Let us consider the energy dissipation rate. Recall that $\boldsymbol{h}_{\text {eff }}=-\partial w / \partial \boldsymbol{m}$, we find out the dissipation rate has the following form

$$
\frac{\mathrm{d} w}{\mathrm{~d} t}=\frac{\partial w}{\partial \boldsymbol{m}} \cdot \frac{\mathrm{d} \boldsymbol{m}}{\mathrm{d} t}=-\frac{\alpha}{1+\alpha^{2}}\left|\boldsymbol{h}_{\mathrm{eff}} \times \boldsymbol{m}\right|^{2} .
$$

For small damping parameter $\alpha$, short pulse period, or small applied field, the damping term in the LLG equation (11) can be neglected. We note that in real experiments the damping parameter is usually of the order of $10^{-2}$, and the field pulse duration can be adjusted to hundreds of picoseconds, thus providing a very good examples of this zero damping model. The dynamics is described by the following equation:

$$
\frac{\partial \boldsymbol{m}}{\partial t}=-\boldsymbol{m} \times \boldsymbol{h}_{\mathrm{eff}} .
$$

In terms of the polar angle $\theta$ and the azimuthal angle $\phi$, the energy density reads

$$
\begin{aligned}
w= & \frac{1}{2} \cos ^{2} \theta-\frac{1}{2} K \sin ^{2} \theta \cos ^{2} \phi \\
& -h_{x} \sin \theta \cos \phi-h_{y} \sin \theta \sin \phi-h_{z} \cos \theta,
\end{aligned}
$$

and the equations of motion are

$$
\dot{\theta}=-\frac{1}{\sin \theta} \frac{\partial w}{\partial \phi}, \quad \dot{\phi}=\frac{1}{\sin \theta} \frac{\partial w}{\partial \theta} .
$$

For simplicity we consider field pulses with zero rise and fall times. That is, the applied field remains constant for a pulse duration of $T$. We then define the problem as follows. Assume initially the magnetization is along the $+\hat{x}$ direction at $\theta=\pi / 2, \phi=0$, denoted by $\boldsymbol{m}_{0}$. By applying a field pulse we expect the magnetization to switch to the $-\hat{x}$ direction at $\theta=\pi / 2, \phi=\pi$, denoted by $\boldsymbol{m}_{1}$. In order to achieve this both field strength and pulse duration have to satisfy certain requirement. Here we will mainly consider the field strength requirement.

\section{ZERO DAMPING: SWITCHING FIELD IN $y-z$ PLANE}

We first study the $\alpha=0$ case. In this case, the magnetization cannot relax to a local minimum by dissipating 


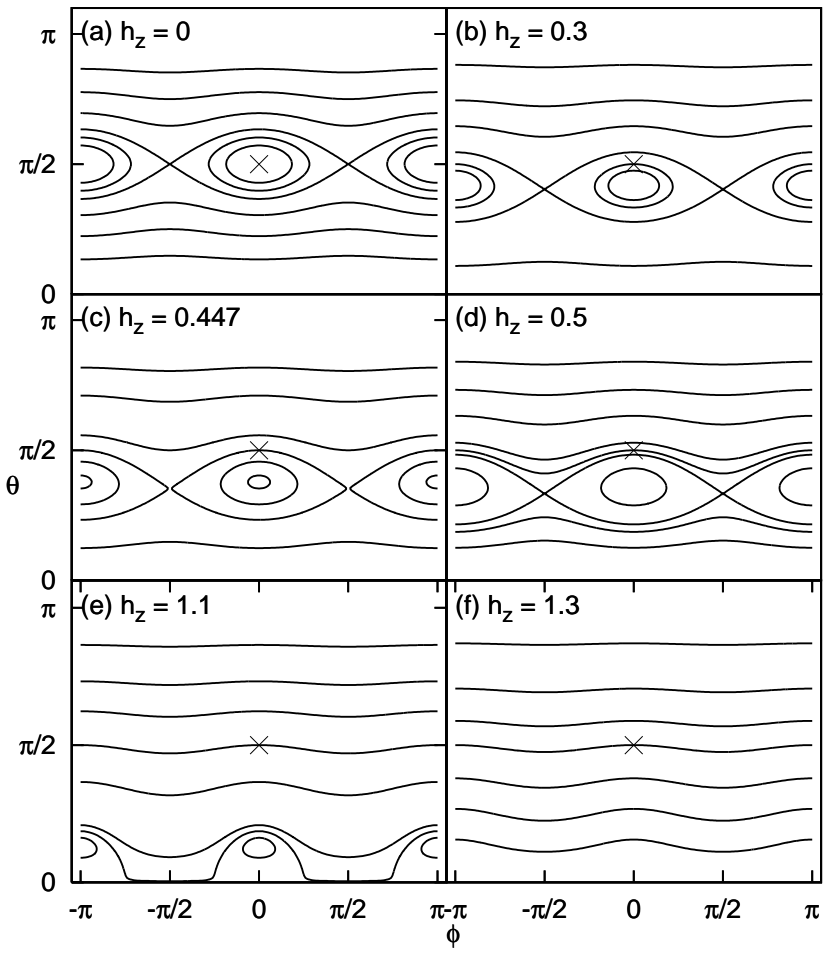

FIG. 2: The phase space at $K=0.2$ for different $h_{z}$. Point $\mathrm{X}$ denotes the initial state $\boldsymbol{m}_{0}$. In (a) $h_{z}=0, \boldsymbol{m}_{0}$ is a local minimum. For $0<h_{z}<1, \boldsymbol{m}_{0}$ gradually moves out from the localized region as shown in (b)-(d). In (e) $1<h_{z}<1+K$, the two saddle points collide at the north pole, end up with only one saddle point. In (f) all trajectories are unlocalized in the range $h_{z}>1+K$.

energy, therefore the initial state $\boldsymbol{m}_{0}$ and the final state $\boldsymbol{m}_{1}$ must be connected by a constant energy curve during the application of the field pulse. This requirement leads to $\boldsymbol{h} \cdot\left(\boldsymbol{m}_{1}-\boldsymbol{m}_{0}\right)=0$; the applied field must be in the $y$-z plane.

The phase space without applied field is shown in Fig. 2]. The system has (a) two minima at $\theta=\pi / 2$, $\phi=0, \pi$, (b) two maxima at $\theta=0, \pi$ ( $\phi$ has no definition), and (c) two saddle points at $\theta=\pi / 2, \phi= \pm \pi / 2$. The phase space is divided by the separatrix (trajectories that join the saddle points) into four regions. Inside the separatrix there are two regions, where the motion is periodic and localized around the corresponding minima. In a dissipative system these two regions are called basin of attraction for these two minima. Outside the separatrix, in the top and bottom, the motion is unlocalized, i.e., the magnetization can move from the $+\hat{x}$ direction to the $-\hat{x}$ direction. Generally speaking, in a precessional switching the function of the applied field is to tilt the phase space structure and drag the initial state $\boldsymbol{m}_{0}$ out of the localized region so the magnetization can precess globally.

To better illustrate this idea, we consider two special cases in which the applied field is along the $\hat{z}$ direction and $\hat{y}$ direction, respectively.

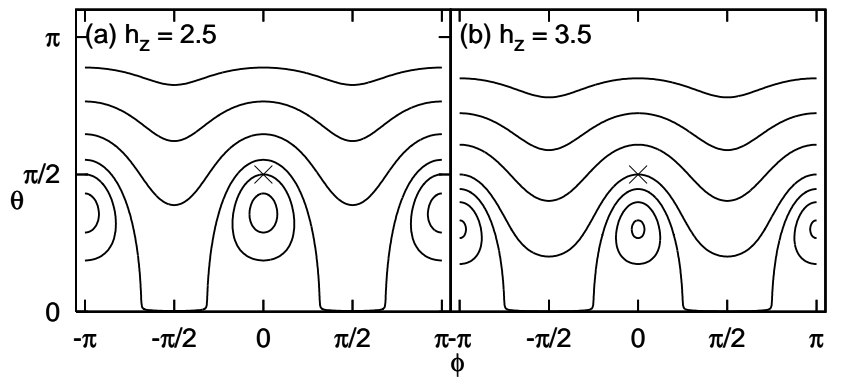

FIG. 3: The phase space at $K=5$ for different $h_{z}$. Point X denotes initial state $\boldsymbol{m}_{0}$. In (a) $1<h_{z}<(1+K) / 2, \boldsymbol{m}_{0}$ stays in the localized region. In (b) $(1+K) / 2<h_{z}<1+K, \boldsymbol{m}_{0}$ is on a unlocalized trajectory.

In case (i), the field $\boldsymbol{h}$ is applied along the $\hat{z}$ direction. The phase space at $K<1$ is shown in Fig. 2 p-f. In the range of $h_{z}<1$, the phase space has the same topological structure as $h_{z}=0$. There are (a) two minima at $\theta=\arccos \left[h_{z} /(1+K)\right], \phi=0, \pi$, (b) two maxima at $\theta=0, \pi$, and (c) two saddle points at $\theta=\arccos h_{z}$, $\phi= \pm \pi / 2$. In the range $0<h_{z}<\sqrt{K}$, the initial state $\boldsymbol{m}_{0}$ stays in the central localized region, no magnetization switching will occur (Fig. 2b). If $h_{z}=\sqrt{K}, \boldsymbol{m}_{0}$ is on the separatrix and it could evolve into the other localized region, that is when the switching starts (Fig. 2r). Note that on the separatrix the motion is however very slow when approaching the saddle points.. As shown in Fig. 28, in the range $1<h_{z}<1+K$, the two saddle points at $\theta=\arccos h_{z}, \phi= \pm \pi / 2$ disappear, and $\theta=0$ becomes a saddle point, i.e., the separatrix passes the north pole. Finally for $h_{z}>1+K$ there are only one minimum at $\theta=0$ and one maximum at $\theta=\pi$, all trajectories are unlocalized.

If $K>1$, i.e., the anisotropy of the easy axis is bigger than that of the hard axis, the two wells around the energy minima are so deep that even after $h_{z}$ passes 1 the initial state $\boldsymbol{m}_{0}$ still stays in the localized region, shown in Fig. 37. calculation shows the minimal field strength that causes switching is $h_{z}=(1+K) / 2$, bigger than $\sqrt{K}$.

Now let us consider the case where the field $\boldsymbol{h}$ is applied along the haty direction. The $h_{y}$ field breaks the $y$ axis symmetry. As a consequence, the two saddle points have different energies. The phase space at $K<1$ is shown in Fig. 4. In the range of $0<h_{y}<K$, there are (a) two minima at $\theta=\pi / 2, \phi=\arcsin h_{y} / K, \pi-\arcsin h_{y} / K$, (b) two maxima at $\theta=\arcsin h_{y}, \pi-\arcsin h_{y}, \phi=-\pi / 2$, and (c) two saddle points at $\theta=\pi / 2, \phi= \pm \pi / 2$. As $h_{y}$ increases, the size of localized regions shrinks. The precessional switching occurs at $h_{y}=K / 2$ (Fig. 4b). For $h_{y}>K$, the two minima collide together with one of the saddle points and disappear, a new minima emerges at $\theta=\pi / 2, \phi=\pi / 2$. After $h_{y}$ passes 1 there are only one minimum at $\theta=\pi / 2, \phi=\pi / 2$ and one maximum at $\theta=\pi / 2, \phi=-\pi / 2$. If $K>1$, evolution of the phase space will be different, but the critical condition $h_{y}=K / 2$ still holds. 


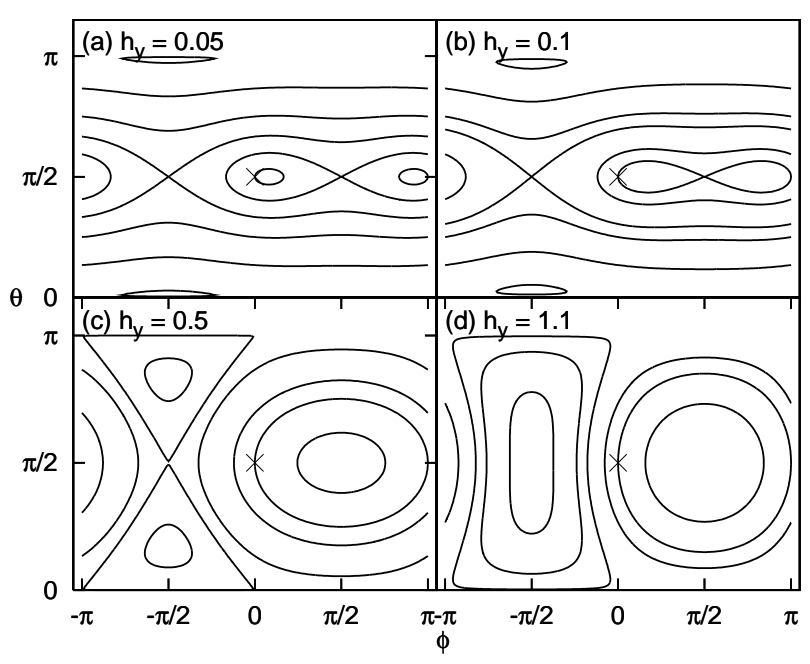

FIG. 4: The phase space at $K=0.2$ for different $h_{y}$. Point $\mathrm{X}$ denotes the initial state $\boldsymbol{m}_{0}$. In (a) and (b) $0<h_{y}<K$. At $h_{y}=K / 2, \boldsymbol{m}_{0}$ is on the separatrix. In (c) $K<h_{y}<1$. In (d) $h_{y}>1$.

From the above discussion, we can see that precessional switching occurs during the transition of trajectories around the initial state $\boldsymbol{m}_{0}$ from localized to unlocalized. In other words, this is when $\boldsymbol{m}_{0}$ moves from inside to outside the separatrix, so the critical field is the one that makes $\boldsymbol{m}_{0}$ on the separatrix. Since points in the phase space move along trajectories of constant energy, the critical condition can be also stated as that the energy of initial state equals the energy of saddle points (but we need check if they are on the same trajectory). We observe that this situation usually happens before collision of two fixed points, which is the critical condition for the SW type reversal. This explains the smaller switching field in precessional switching.

Now we consider the general case in which the field $\boldsymbol{h}$ is applied in the $y-z$ plane at an arbitrary angle. The energies of the initial state $\boldsymbol{m}_{0}$ and final state $\boldsymbol{m}_{1}$ are always $-K / 2$. We are looking for saddle points with the same energy. The fixed points are given by

$$
-\sin \theta \cos \theta\left(1+K \cos ^{2} \phi\right)-h_{y} \cos \theta \sin \phi+h_{z} \sin \theta=0,
$$

$$
K \sin ^{2} \theta \sin \phi \cos \phi-h_{y} \sin \theta \cos \phi=0 .
$$

From Eq. (7b) we see that solutions can be grouped into two categories. The first category includes solutions with $\sin \phi=h_{y} /(K \sin \theta)$. Inserting this expression into Eq. 17a) and solving it gives us fixed points

$$
\cos \theta=\frac{h_{z}}{1+K}, \quad \sin \phi=\frac{h_{y}}{K \sqrt{1-h_{z}^{2} /(1+K)^{2}}},
$$

with the condition

$$
\frac{h_{y}^{2}}{K^{2}}+\frac{h_{z}^{2}}{(1+K)^{2}} \leq 1 .
$$
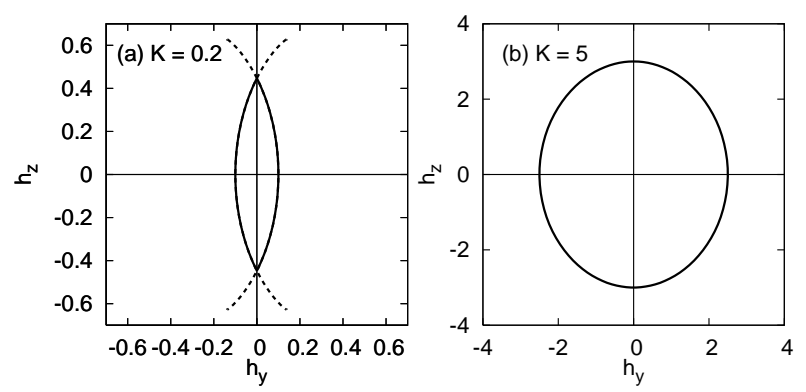

FIG. 5: Critical switching field in the $y-z$ plane at $\alpha=0$ for (a) $K<1$ and (b) $K>1$. The intersections of the curve are in (a) $h_{y}=K / 2, h_{z}=\sqrt{K}$, in (b) $h_{y}=K / 2, h_{z}=(K+1) / 2$. Fields shown in dashed line corresponds to the situation where the initial state has the same energy with one of the saddle points but is not on the separatrix.

In following discussion we assume this condition always holds since we are interested in the minimal field strength. We will see later the result is consistent with this assumption. The energy of the fixed points is

$$
E=-\frac{K}{2}-\frac{1}{2}\left(\frac{h_{y}^{2}}{K}+\frac{h_{z}^{2}}{1+K}\right) .
$$

The energy is always smaller than $-K / 2$ so they are not what we are looking for. Stability analysis shows in fact they are energy minima.

Now we turn to the other category, which includes solutions with $\cos \phi=0$. In this category, fixed points occur on a great circle parametrized by setting $\phi=0$ and letting $\theta$ run from 0 to $2 \pi$. This makes the problem essentially a two-dimensional problem in the $y$ - $z$ plane.

Instead of numerics, this problem can be solved by a geometrical approach, first proposed by Slonczewski in the Stoner-Wohlfarth problem, then extended by Thiaville ${ }^{4.5}$ In essence, it considers the field $\boldsymbol{h}$, instead of the magnetization direction $\boldsymbol{m}$ as the main variable. We write $\boldsymbol{m}=\sin \theta \hat{y}+\cos \theta \hat{z}=(\sin \theta, \cos \theta)$ and its orthogonal vector $\boldsymbol{e}=(\cos \theta,-\sin \theta)$. The energy requirement is

$$
E=\frac{1}{2} \cos ^{2} \theta-\boldsymbol{h} \cdot \boldsymbol{m}=-\frac{K}{2} .
$$

The extremum condition is

$$
\frac{\mathrm{d} E}{\mathrm{~d} \theta}=-\sin \theta \cos \theta-\boldsymbol{h} \cdot \boldsymbol{e}=0 .
$$

Since the system already has two minima at points (8), the saddle point we are looking for has to be the minimum along the $\hat{\theta}$ direction. This leads to

$$
\frac{\mathrm{d}^{2} E}{\mathrm{~d} \theta^{2}}=\sin ^{2} \theta-\cos ^{2} \theta+\boldsymbol{h} \cdot \boldsymbol{m}>0 .
$$

The critical switching field is determined by the intersections of the two lines defined in Eqs. (11) and (12). 


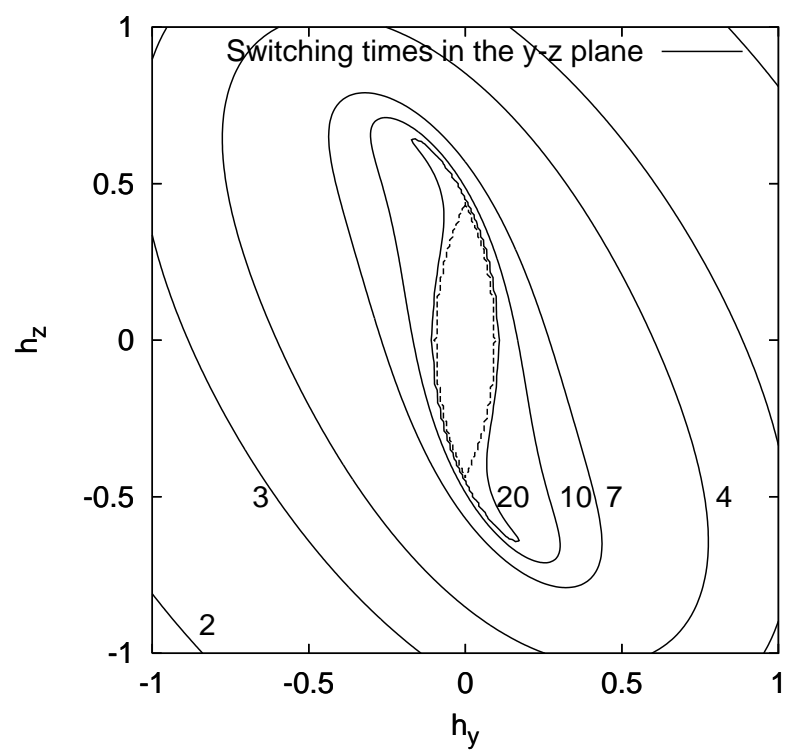

FIG. 6: Switching times contour in the $y$ - $z$ plane for $K=0.2$. The dashed line in the center is the critical switching field. From outside to inside, the switching times are 2, 3, 4, 7, 10,20 , measured in $\left(\gamma M_{s}\right)^{-1}$. For typical configuration it is about $0.01 \mathrm{~ns}$.

We find

$$
\begin{aligned}
& h_{y}=\frac{1}{2} \sin \theta\left(K-\cos ^{2} \theta\right), \\
& h_{z}=\frac{1}{2} \cos \theta\left(1+K+\sin ^{2} \theta\right) .
\end{aligned}
$$

Inserting above expressions into Eq. (13) yields

$$
\frac{\mathrm{d}^{2} E}{\mathrm{~d} \theta^{2}}=1+\frac{1}{2} K-\frac{3}{2} \cos ^{2} \theta>0 .
$$

For $K>1$, the above inequality always holds. For $K<1$ we have $|\cos \theta|<\sqrt{(2+K) / 3}$. However, in this range the critical curve does not form a closed loop, shown in Fig. 5 . The reason is that at nonzero $h_{y}$ the two saddle points have different energies so the trajectory starting from $\boldsymbol{m}_{0}$ may not pass that saddle point. After checking we can remove these "faked" solutions by requiring $|\cos \theta|<\sqrt{K}$.

From Fig. [5] we can see that if $K \gg 1$ the magnitude of critical switching field along any direction in the $y-z$ plane is about the same; for magnetic thin films where $K \ll 1$ then $\hat{y}$ direction is the best choice to apply small field. We also calculate the switching time for $K<1$ by numerically integrating Eq. (6). The equi-time contours are plotted in Fig. 6] As the magnitude of fields increases, the switching time decreases quickly from the center.

\section{SMALL DAMPING: SWITCHING FIELD IN $x-y$ PLANE}

In this section we study the small damping case. As finding the exact analytical solution to the equation of magnetization motion including damping is a formidable task, we turn to study the limiting case, where $\alpha$ is small enough so that we can still treat the problem as a Hamiltonian problem and $\alpha$ is also big enough so that after the application of the field pulse the magnetization can relax to the local minimum in finite time. Because of damping the energies of the initial and final state need not to be the same, a nonzero field component along the $\hat{x}$ direction is allowed.

We choose to apply the field in the $x-y$ plane. By this way, we can compare our result with the SW model. The basic procedure is the same as last section: we will search the saddle points with the same energy of the initial state, then map to the whole switching field.

It can be seen from Fig. 4 that the two fixed points that originate from the south and north pole do not involve in this precessional switching, so the saddle point we are looking for is on the $x-y$ plane too. Let $\theta=\pi / 2$, then the problem turns into a two-dimensional problem in the $x-y$ plane. We write $\boldsymbol{m}=\cos \phi \hat{x}+\sin \phi \hat{y}=(\cos \phi, \sin \phi)$ and its orthogonal vector $\boldsymbol{n}=(-\sin \phi, \cos \phi)$. The applied field is $\boldsymbol{h}=\left(h_{x}, h_{y}\right)$.

The energy requirement is

$$
E=-\frac{K}{2} \cos ^{2} \phi-\boldsymbol{h} \cdot \boldsymbol{m}=-\frac{K}{2}-h_{x} .
$$

The extremum condition is

$$
\frac{\mathrm{d} E}{\mathrm{~d} \phi}=K \cos \phi \sin \phi-\boldsymbol{h} \cdot \boldsymbol{n}=0 .
$$

Solving above equations gives us the switching field in the $x-y$ plane:

$$
\begin{aligned}
& h_{x}=-\frac{K}{2} \cos \phi(1+\cos \phi), \\
& h_{y}=\frac{K}{2} \sin \phi(1-\cos \phi) .
\end{aligned}
$$

In order to check the stability of the corresponding fixed point we carry out the standard routine. With $h_{x}, h_{y}$ taking the above form, the second derivatives of $w$ are

$$
\begin{aligned}
\frac{\partial^{2} w}{\partial \theta^{2}} & =1+\frac{K}{2}(1-\cos \phi) \\
\frac{\partial^{2} w}{\partial \phi^{2}} & =K(\cos \phi-1)\left(\cos \phi+\frac{1}{2}\right), \\
\frac{\partial^{2} w}{\partial \theta \partial \phi} & =0
\end{aligned}
$$

It is obvious that $\phi$ has to in the range $0<\phi<\frac{3}{4} \pi$ (upper branch in Fig. 7) or $-\frac{3}{4} \pi<\phi<0$ (lower branch in Fig. (7) to make the fixed point a saddle point. Again we still need to check if the saddle point and the initial state are on the same trajectory. We require both $h_{x}$ and $h_{y}$ are monotonous function of $\phi$ in the upper and lower branches, which narrows the range to $-\frac{2}{3} \pi<\phi<\frac{2}{3} \pi$.

To compare with the switching field in the SW model, which takes the form $\boldsymbol{h}=\left(-\cos ^{3} \phi, \sin ^{3} \phi\right)$, we plot both 


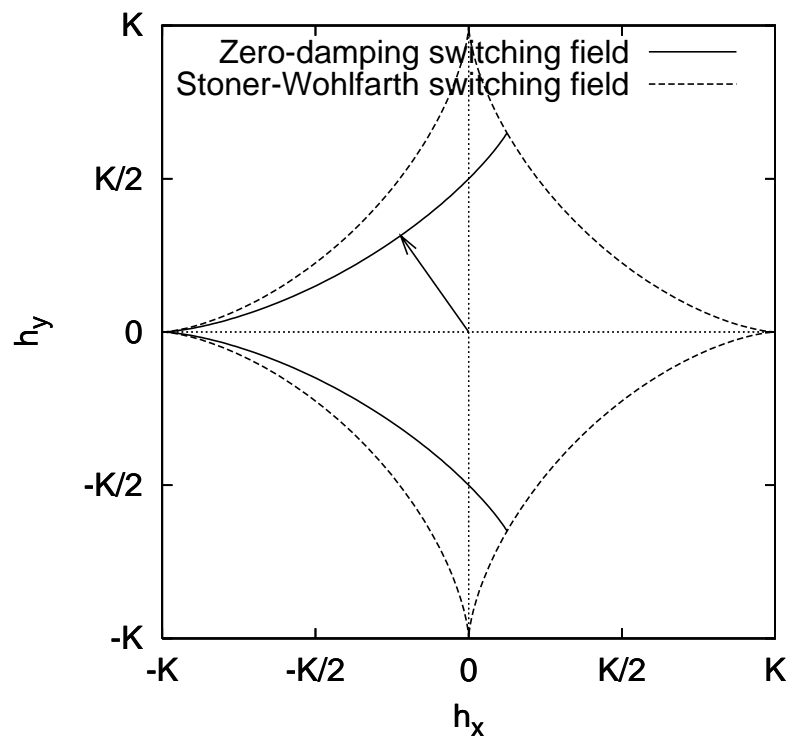

FIG. 7: Critical switching field in the $x-y$ plane for switching from the $\hat{x}$ direction to the $-\hat{x}$ direction. The solid line shows the switching field of precessional switching at zero damping limit. The dashed line is the switching field of the StonerWohlfarth model. For completion we also draw the right half of the SW astroid. Arrow indicates the minimal field strength.

fields in Fig. 17 It is clear that precessional switching can occur well below the SW limit. Surprisingly, magnetic switching is possible for a field with a positive $x$ compo- nent. Although in this case the minimal field strength is $0.38 K(\cos \phi=1 / 3)$, considering the long relaxation time it is still better to apply the field mainly along $\hat{y}$ direction with a small $x$ component to compensate the energy loss during the motion.

\section{SUMMARY}

The critical switching field of a monodomain magnetic object with biaxial anisotropy has been studied. In particular, switching fields perpendicular to the easy and hard axes are calculated using the geometrical method. It is found that in the limit of zero damping, applying field along the medium axis is good for both small fields and fast switching times.

This method can be generalized for magnetic particles with a higher order anisotropy if one follows the same steps outlined in this paper. The two-dimensional model may not be sufficient because one needs to consider more fixed points. A more complicated three-dimensional model would then be required.

\section{Acknowledgments}

We thank the NSF NIRT program (Grant No. DMR0404252) for support of this work.
* Electronic address: dxiao@physics.utexas.edu

1 B. Hillebrands and K. Ounadjela, eds., Spin Dynamics in Confined Magnetic Structures I (Springer-Verlag, Berlin, 2001).

2 B. Hillebrands and K. Ounadjela, eds., Spin Dynamics in Confined Magnetic Structures II (Springer-Verlag, Berlin, 2003).

3 E. C. Stoner, F.R.S. and E. P. Wohlfarth, Phil. Trans. Roy. Soc. London A240, 599 (1948), reprinted in IEEE Trans. Magn. 27, 3475 (1991).

4 A. Thiaville, J. Mag. Mag. Mater 182, 5 (1998).

5 A. Thiaville, Phys. Rev. B 61, 12221 (2000).

${ }^{6}$ C. H. Back, D. Weller, J. Heidmann, D. Mauri, D. Guarisco, E. L. Garwin, and H. C. Siegmann, Phys. Rev. Lett. 81, 3251 (1998).

7 C. H. Back, R. Allenspach, W. Weber, S. S. P. Parkin, D. Weller, E. L. Garwin, and H. C. Siegmann, Science 285, 864 (1999).
8 M. Bauer, J. Fassbender, B. Hillebrands, and R. L. Stamps, Phys. Rev. B 61, 3410 (2000).

9 Y. Acremann, C. H. Back, M. Buess, D. Pescia, and V. Pokrovsky, Appl. Phys. Lett. 79, 2228 (2001).

10 J. Miltat, G. Albuquerque, and A. Thiaville, in Spin Dynamics in Confined Magnetic Structures I, edited by B. Hillebrands and K. Ounadjela (Springer-Verlag, Berlin, 2001), pp. 1-33.

11 H. W. Schumacher, C. Chappert, P. Crozat, R. C. Sousa, P. P. Freitas, J. Miltat, J. Fassbender, and B. Hillebrands, Phys. Rev. Lett. 90, 17201 (2003).

12 H. W. Schumacher, C. Chappert, R. C. Sousa, P. P. Freitas, and J. Miltat, Phys. Rev. Lett. 90, 17204 (2003).

${ }^{13}$ L. He, W. D. Doyle, and H. Fujiwara, IEEE. Trans. Magn. 30, 4086 (1994).

14 L. He and W. D. Doyle, J. Appl. Phys. 79, 6489 (1996). 